\title{
The Mediator Role of Depression and Anxiety in the Relationship between Cognitive Emotional Regulation and Self-care in Type 2 Diabetes
}

\author{
Somayeh Ramesh (MSc) ${ }^{1, *}$, Mona Ghazian (MSc)르 Amin Rafiepoor (PhD) ${ }^{3}$, Ali Reza \\ Safari $(\mathbf{P h D})^{4}$ \\ ${ }^{1}$ PhD student of Health psychology, Department of Psychology, Faculty of Psychology and Education, University of Tehran, \\ Tehran, Iran \\ ${ }^{2}$ MSc Clinical Psychology, Department of Psychology, Faculty of Psychology and Education, University of Tehran, Tehran, \\ Iran \\ ${ }^{3}$ Assistant Professor, Department of Psychology, Payame Noor University, Tehran, Iran \\ ${ }^{4}$ MSc Clinical Psychology, Department of Psychology, Faculty of Psychology and Education, Islamic Azad University \\ Science and Research Branch, Tehran, Iran
}

* Corresponding Author: Somayeh Ramesh, Department of Psychology, Faculty of Psychology and Education, University of Tehran, Tehran, Iran. Tel: 09133735417; Email: Ramesh_somayeh@yhoo.com

\begin{tabular}{|c|c|}
\hline & Abstract \\
\hline $\begin{array}{l}\text { Received: 29/04/2018 } \\
\text { Accepted: 23/06/2018 }\end{array}$ & \multirow[b]{2}{*}{$\begin{array}{l}\text { Background and Objectives: Diabetes is one of the most common chronic } \\
\text { diseases and requires self-care. The purpose of this study was to investigate } \\
\text { the mediator role of depression and anxiety in the relationship between } \\
\text { cognitive emotional regulation and self-care in type } 2 \text { diabetic patients. } \\
\text { Materials and Methods: } 108 \text { patients with type } 2 \text { diabetes ( } 57 \text { women, } 51 \\
\text { males) participated in this study. The patients were selected through } \\
\text { targeted sampling from those referring to health centers in Tehran in } 2016 \text {. } \\
\text { The participants completed the } 21 \text { st-DASS Questionnaire, Cognitive } \\
\text { Emotion Regulation Questionnaire (CERQ), and the Self-Care behaviors } \\
\text { Questionnaire (SDSCA). Data were analyzed with descriptive and } \\
\text { inferential statistics by using SPSS software version } 16 \text {. } \\
\text { Results: The results of this study showed that self-care and adaptive } \\
\text { strategies of cognitive emotional regulation with anxiety, have a significant } \\
\text { negative correlation and there is a significant positive correlation between } \\
\text { non-adaptive strategies of cognitive emotion regulation and anxiety } \\
\text { (P<0.01). The results of path analysis also indicated the mediator role of } \\
\text { anxiety in the relationship between cognitive emotion regulation and self- } \\
\text { care in patients with type } 2 \text { diabetes. } \\
\text { Conclusions: According to the result, in the field of self-care in diabetic } \\
\text { patients, it is necessary to consider psychological interventions to regulate } \\
\text { emotions and reduce depression and anxiety. }\end{array}$} \\
\hline $\begin{array}{l}\text { How to Cite this Article: } \\
\text { Ramesh S, Ghazian M, Rafiepoor } \\
\text { A, Safari AR. The Mediator } \\
\text { Role of Depression and Anxiety } \\
\text { in the Relationship between } \\
\text { Cognitive Emotional Regulation } \\
\text { and Self-care in Type } 2 \text { Diabetes. } \\
\text { Pajouhan Scientific Journal. } \\
\text { 2018; 16(4): 37-45. DOI: } \\
\text { 10.29252/psj.16.4.37 }\end{array}$ & \\
\hline & Keywords: Anxiety; Depression; Emotional Regulation; Self-care \\
\hline
\end{tabular}


doi): $10.29252 /$ psj.16.4.37

\section{نقش واسطهاى افسردىى و اضطراب در رابطه بين تنظيم شناختى هيجان و خودمر اقبتى در مبتلايان به ديابت نوع دو استرد}

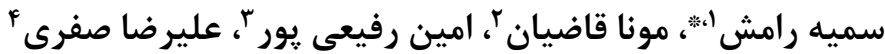

' دانشجوى دكترى تخصصى روانشناسى سلامت، كروه روانشناسى، دانشكده روانشناسى و علوم تربيتى، دانشعاه تهران، تهران، ايران

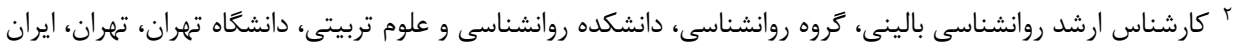

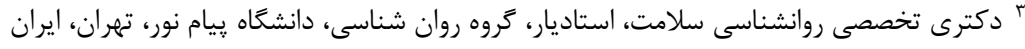

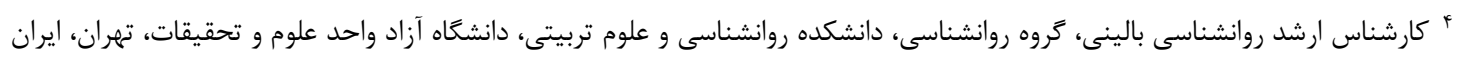
* نويسنده مسئول: سميه رامش، كروه روانشناسى، دانشكده روانشناسى و علوم تربيتى، دانشعاه تهران، تهران، ايران. تلفن: Ramesh_somayeh@yhoo.com

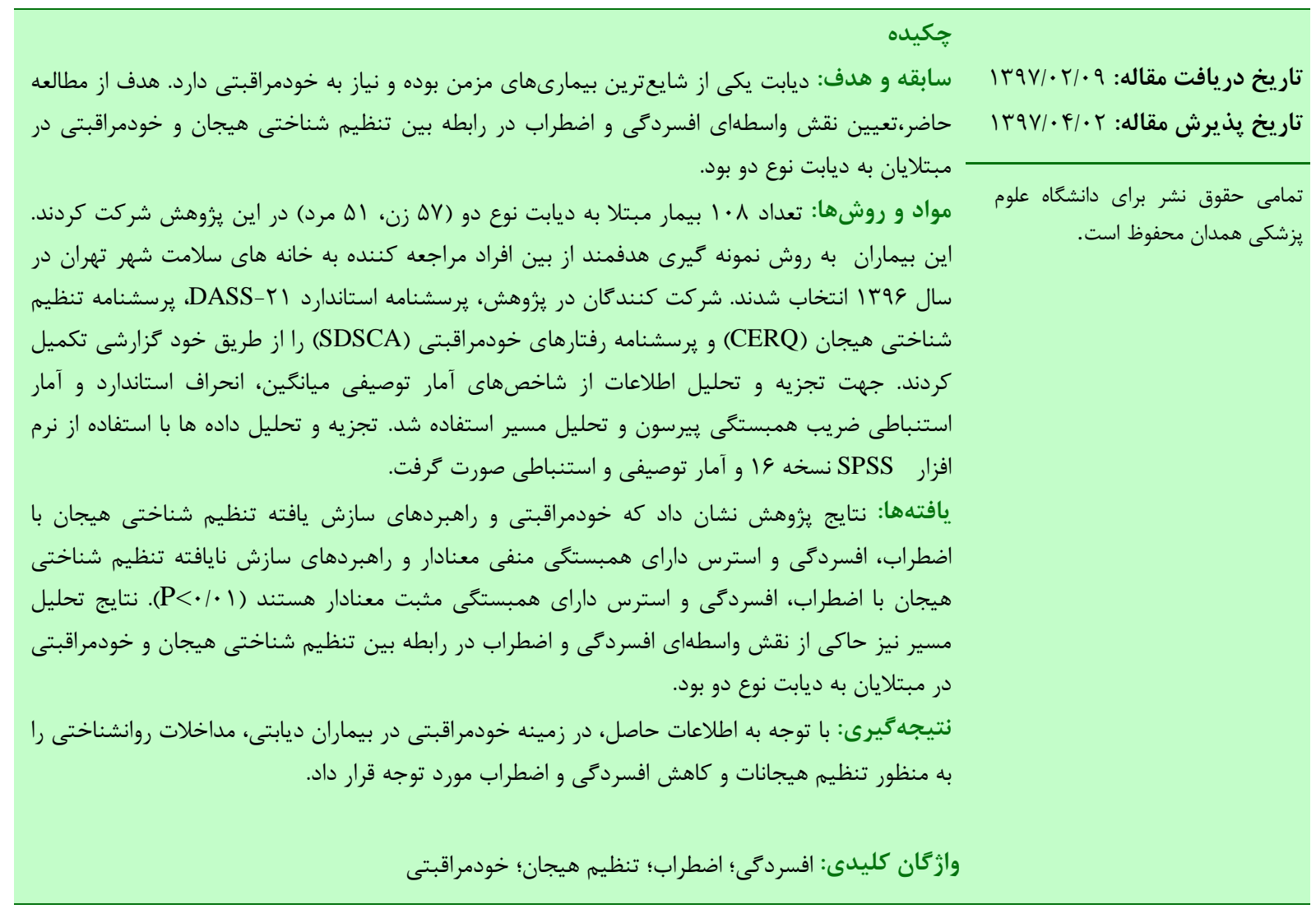

مقلدمه

جمله يا بوده و اميد به زندگى در بيماران ديابتى له تا • ا سـال

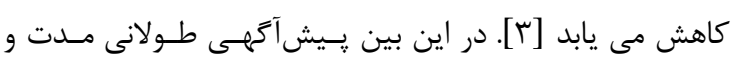

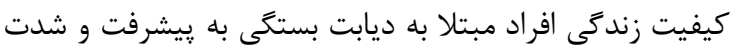

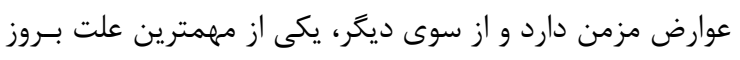

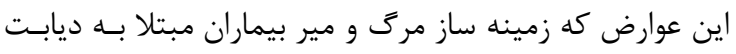

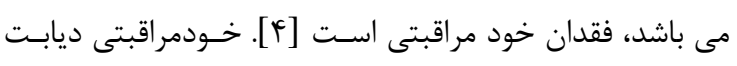

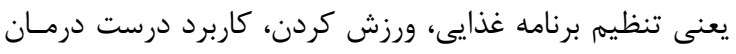

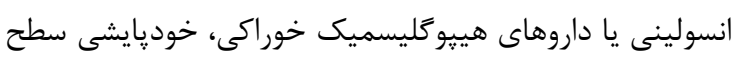

ديابت به عنوان يكى بيمارى مزمن با آسيب، مرك و ميـر و

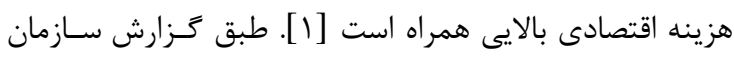

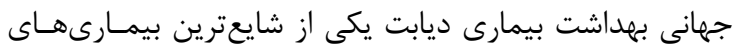

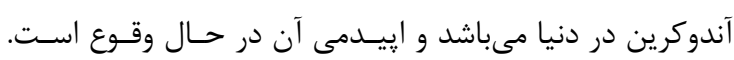

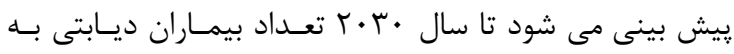

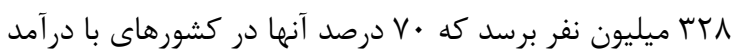

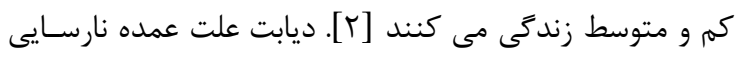

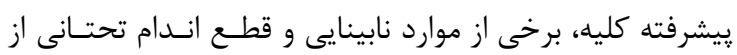




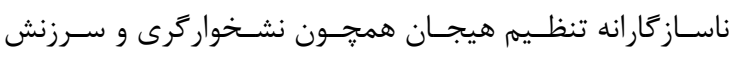

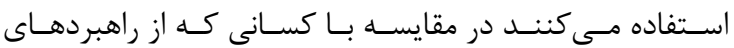

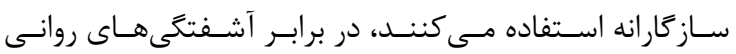

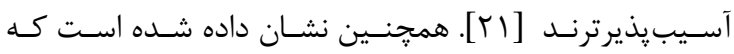

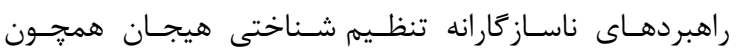

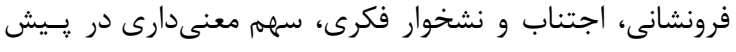

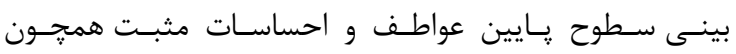

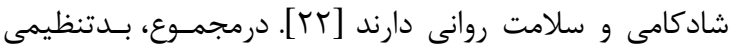

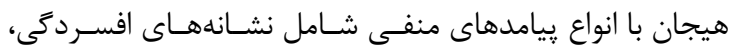

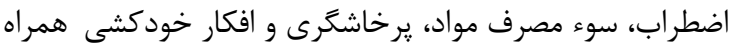

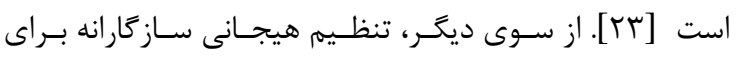

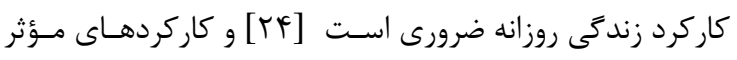

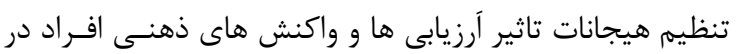

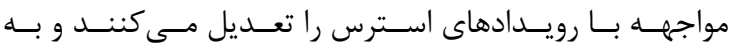
واكنشهاى شناختى، انخيزشى و رفتارى مناسب و مورد نياز در

جنين شرايطى منجر مى شود [Tهائ.

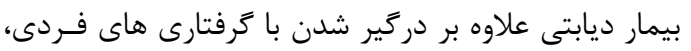

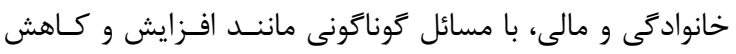

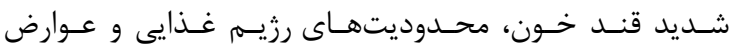

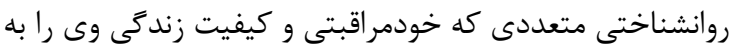

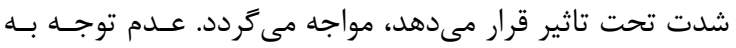

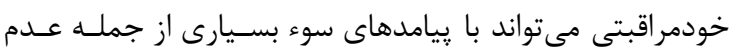

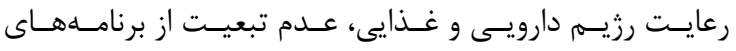

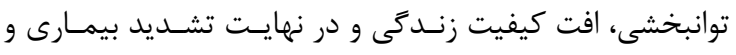

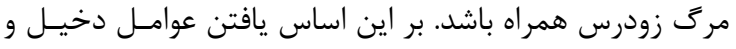

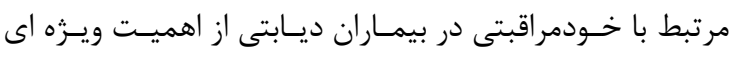
برخوردار است و مطالعه حاضر در همين راستا و با دو هدف: (1) ) تعيين رابطه تنظيم شناختى هيجان با خودمراقبتى در بيمـاران

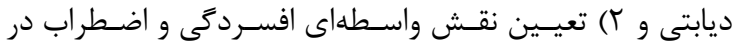

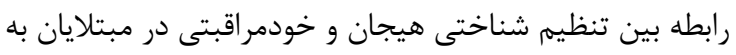
ديابت نوع دو انجام گرفت نئ.

\section{مواد و روشها}

يزوهش حاضر يك مطالعه توصسيفى تحليلـى بـود. جامعـهـ

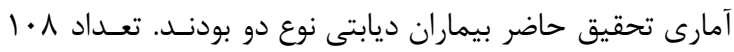

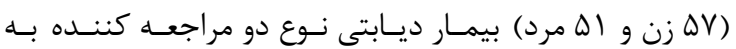

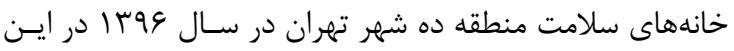

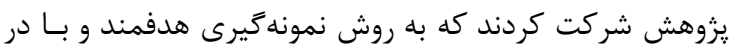

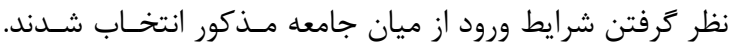

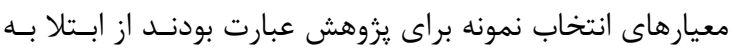

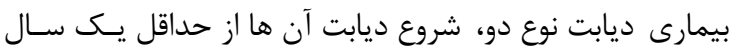

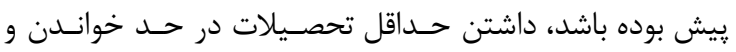

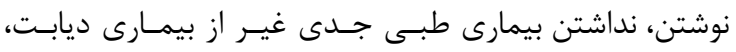

قند خون و مراقبت از پاها (به عنوان پايه كنترل و پيشگيرى از

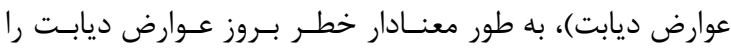
كاهش مى دهد [Y]. تحقيقات نشان داده است كه خـودمراقبتى دئى

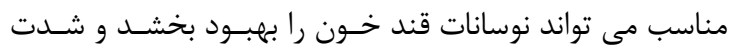

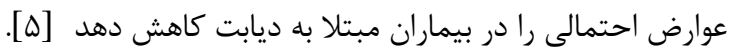

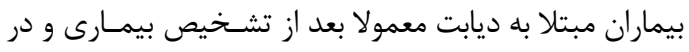

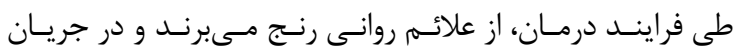

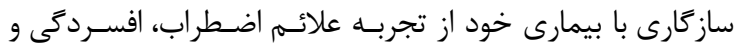

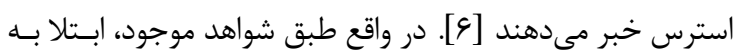

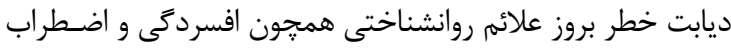

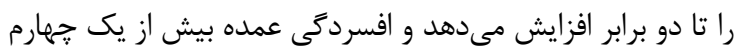

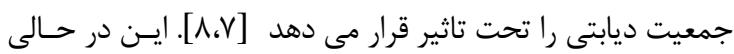

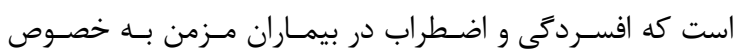

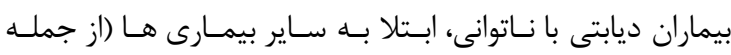

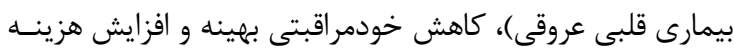

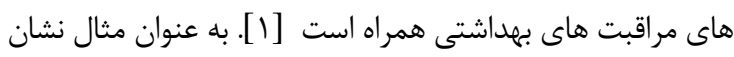

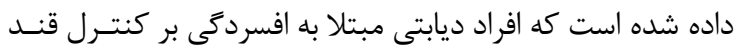

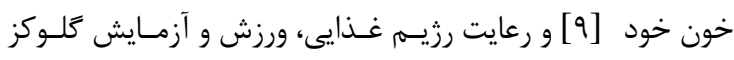

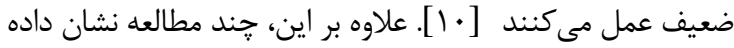

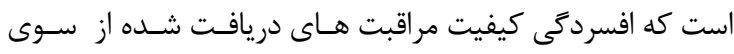
افراد مبتلا به ديابت را كاهش مى دهد [11)] از سوى ديكر، به نظر مىرسد كه خودتنظيمى هيجانى نيـز بر خودمراقبتى در ديابت نقش دارد و اين را به واسطه هيجانات

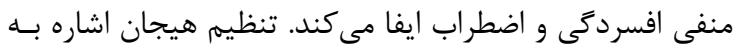

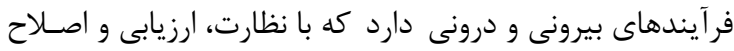

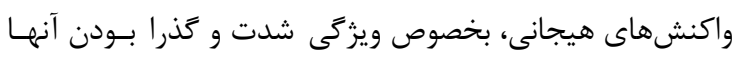

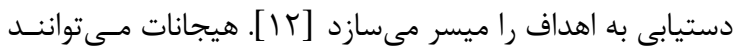

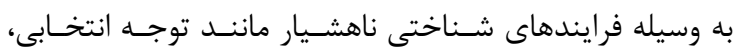

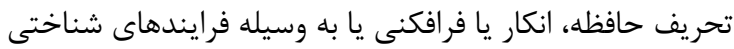

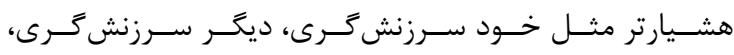

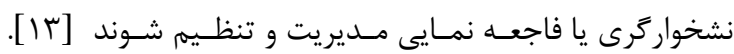
تنظيم هيجان نوع خاصى از خود كنترلى [If

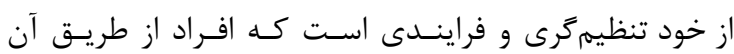

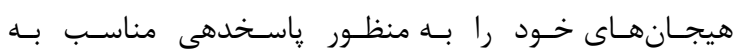

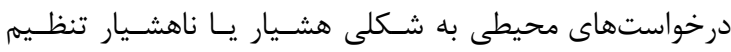

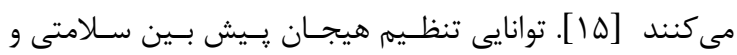

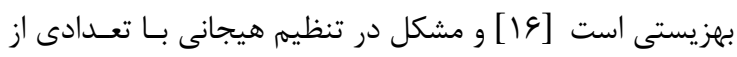

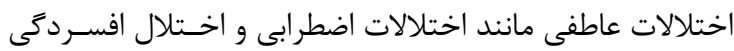

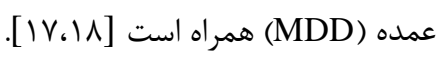
راهبردهاى تنظيم هيجان مى توانند سازكًارانه يا ناساز كارانه

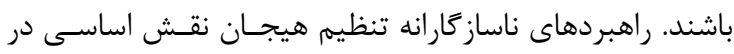

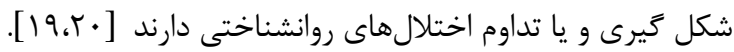

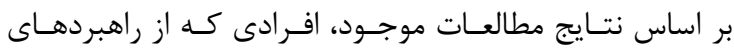


توسط Garnefski N, Kraaij [11 G تدوين شده است و جهت

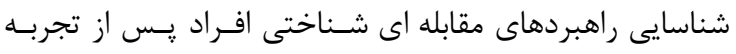

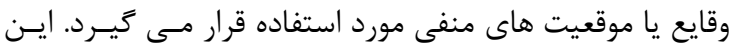

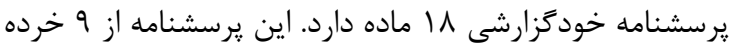

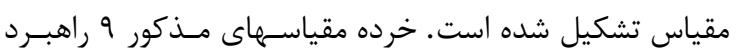

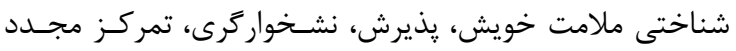

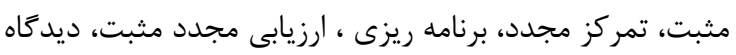

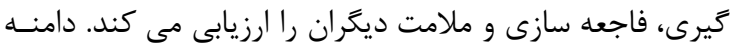

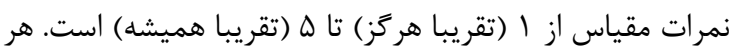

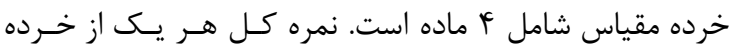
مقياس ها از طريق جمع كردن نمره ماده ها به دست مى مي آيـد.

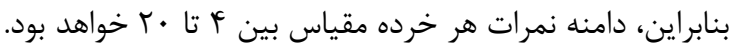

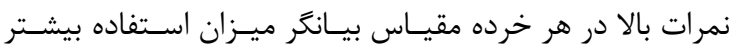

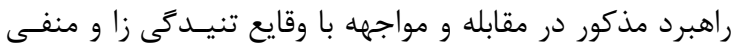

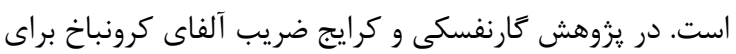

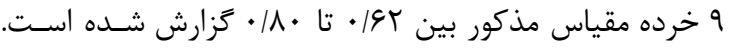

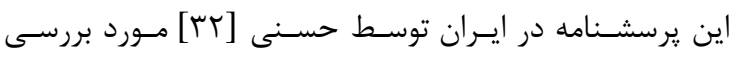

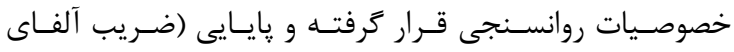

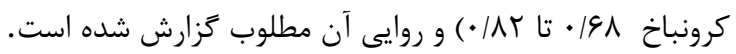

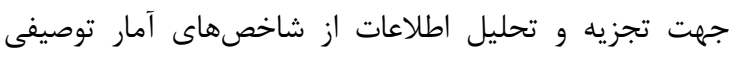

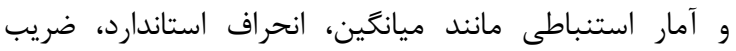

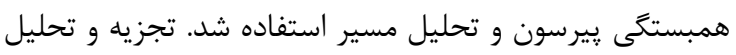

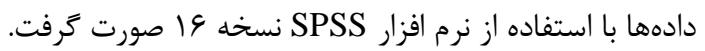

\section{بافتهن.}

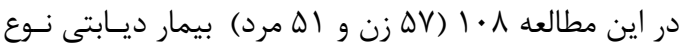

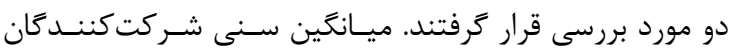

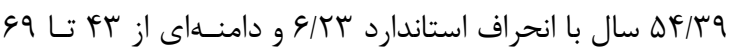

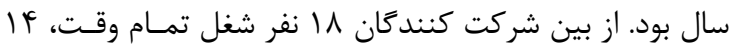

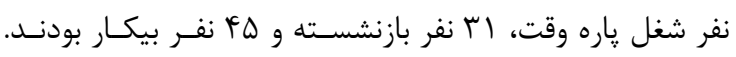

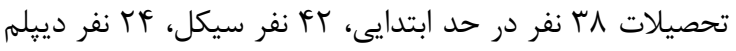

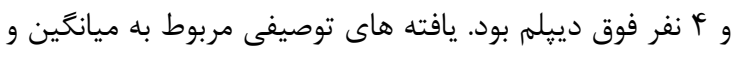

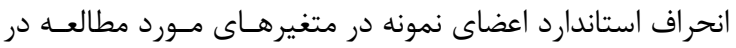

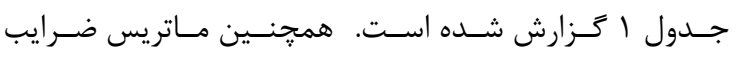
همبستخى متغيرها نيز در جدول ا نشان داده شده است. نتايج

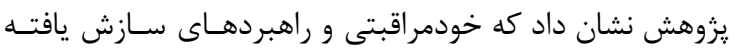

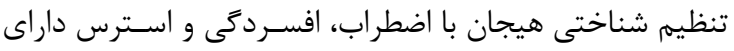

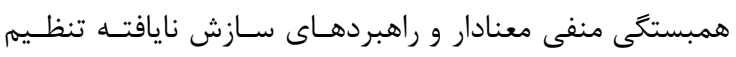

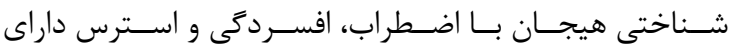

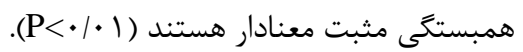

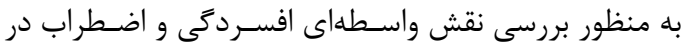
رابطه بين تنظيم شناختى هيجان و خود مراقبتـى در مبتلايسان

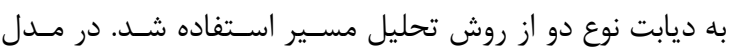

نداشتن اختلال عمده روانيزشكى و عدم وابستخى به مواد. يس از

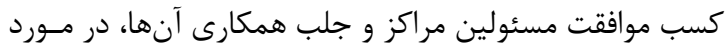

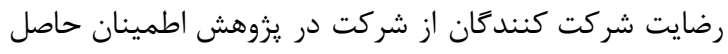

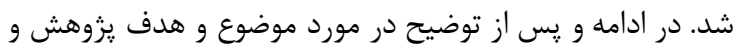

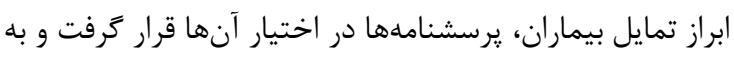

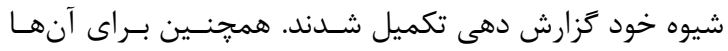

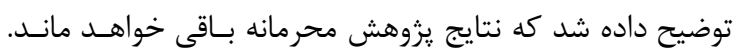

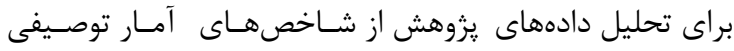

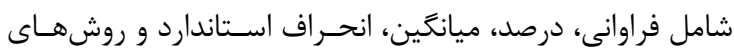

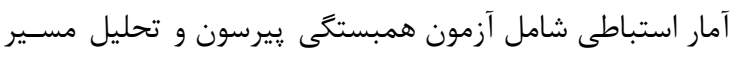

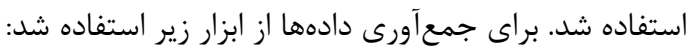

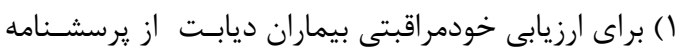

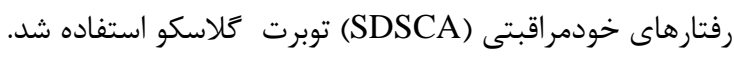

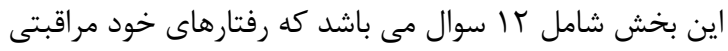

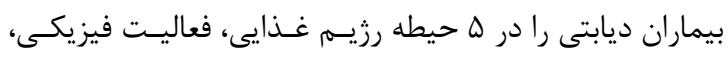

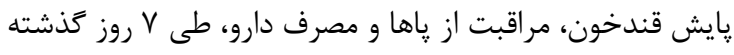

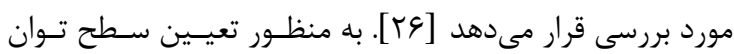

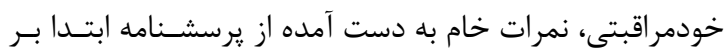

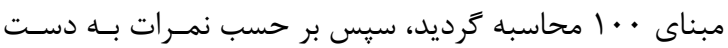

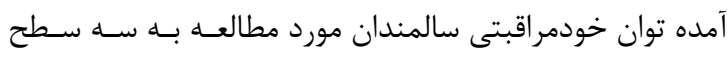

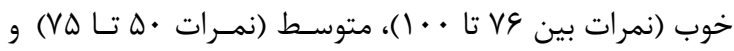

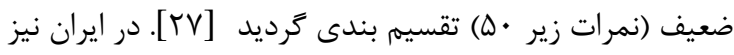

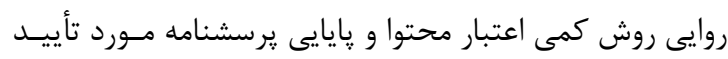

قرار كرفته است [rᄉ].

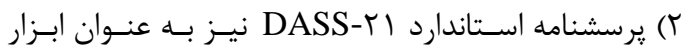

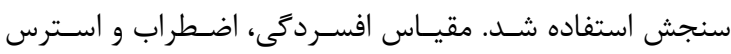

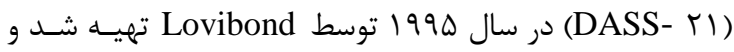

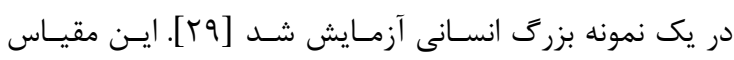

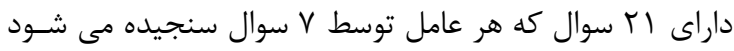

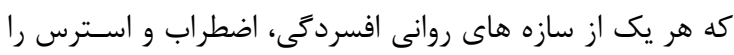

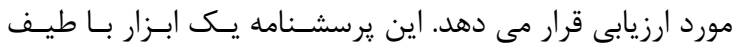
קهار قسمتى است كه گزينه هاى آن از صفر تا سه نمره كذارى

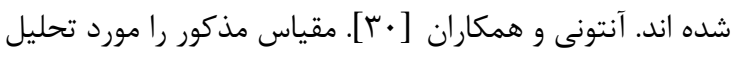

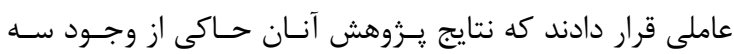

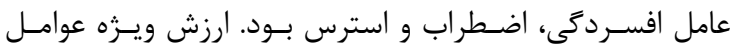

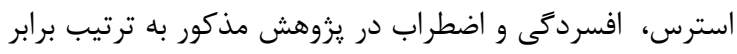

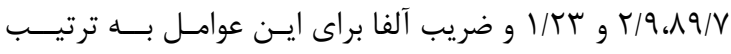

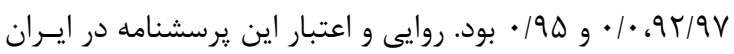

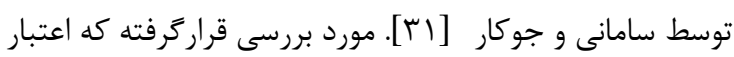

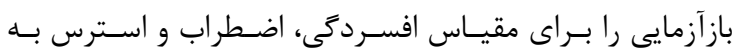

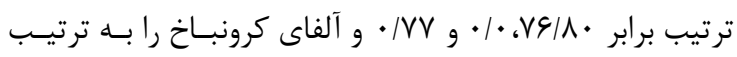

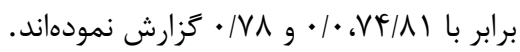

r) يرسشنامه تنظيم شناختى هيجان (CERQ) - فرم كوتاه 
جدول ا: ميانكين، انحراف استاندارد و ضرايب همبستگى خودمراقبتى، راهبردهاى سازش يافته و نايافته تنظيم هيجان، اضطراب، افسردگى و استرس

\begin{tabular}{|c|c|c|c|c|c|c|c|}
\hline$\Delta$ & p & $r$ & r & 1 & SD & $\mathbf{M}$ & 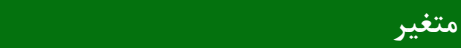 \\
\hline & & & & 1 & $18 / 1 \pi$ & $01 / 91$ & 1- خودمراقبتى \\
\hline & & & 1 & $\cdot / r \mu^{* * * * *}$ & $10 / 91$ & $r \Delta / \Delta V$ & ץ- راهبردهاى سازش يافته تنظيم هيجان \\
\hline & & 1 & $\cdot / \cdot V$ & $-\cdot / \Gamma q^{* * * *}$ & $\Lambda / \Gamma \Delta$ & $r T / T H$ & ץ- راهبردهاى سازش نايافته تنظيم هيجان \\
\hline & 1 & $\cdot / \Gamma V^{\text {米䖭 }}$ & $-\cdot|r|^{* * m}$ & $-\cdot \mid \Delta Y^{* * m}$ & $4 / 19$ & $\Lambda / \Delta 1$ & F اضط - اضراب \\
\hline 1 & $\cdot / q^{* * * *}$ & $\cdot / 1 \pi$ & $-\cdot / r V^{* * *}$ & $-\cdot / 4 \Lambda^{* * *}$ & $r / 9 \Lambda$ & $\Lambda / r V$ & 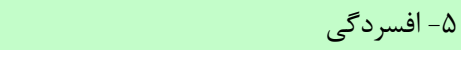 \\
\hline 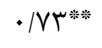 & 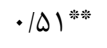 & $\cdot / 11$ & $-\cdot / \mathbb{F} \Delta^{\text {券券 }}$ & $-\cdot|\uparrow|^{* * * m}$ & $\Delta / \mathcal{F}$ & $9 / 91$ & 9- استرس \\
\hline
\end{tabular}

جـدول r ضـرايب اسـتاندارد و غيـر اسـتاندارد مسـيرهاى

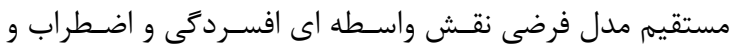

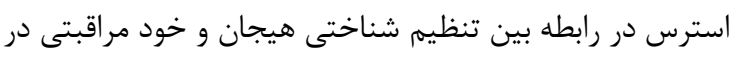

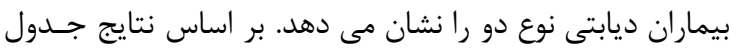

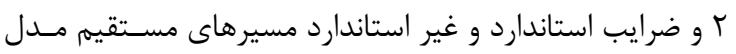

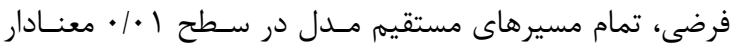

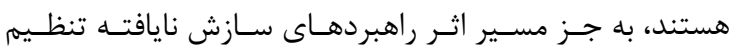

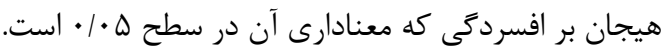

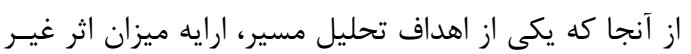

مستقيم متغيرهاى مستقل بر متغيرهاى وابسته است، اثـر غيـر

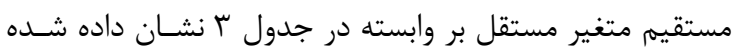
است. همانطور كه مى تـوان مشــاهده كـرد، اثـر غيـر مسـتقيم

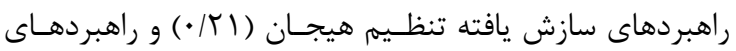

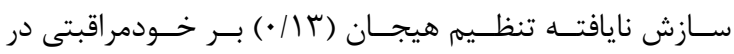

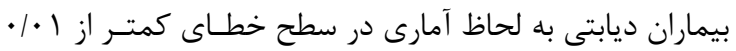

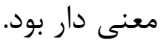
جدول f شاخص هاى برازش مدل فرضى را نشان مى دهد.
مفهومى فرض مى شود كه راهبردهاى تنظيم شناختى هيجـان

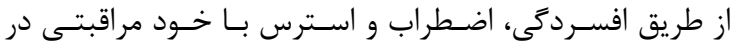

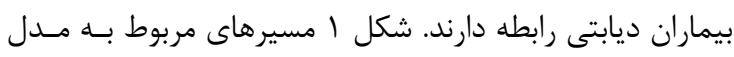

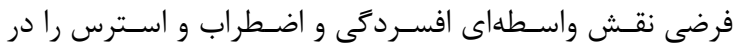

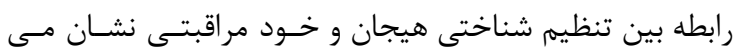

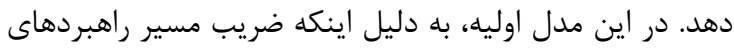

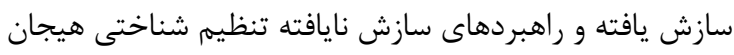

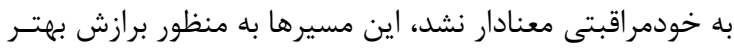

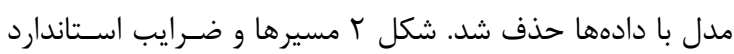

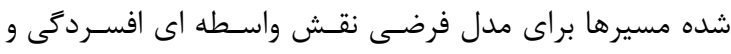

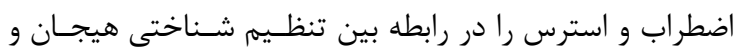

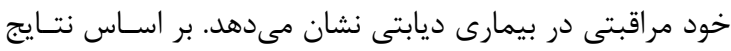

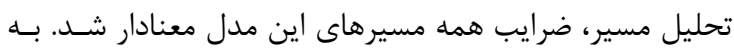

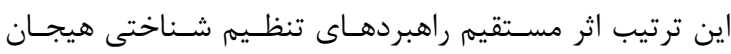

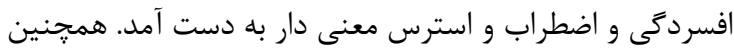

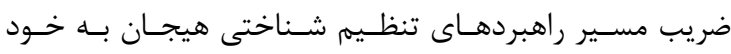
مراقبتى در بيماران ديابتى معنى دار بود.

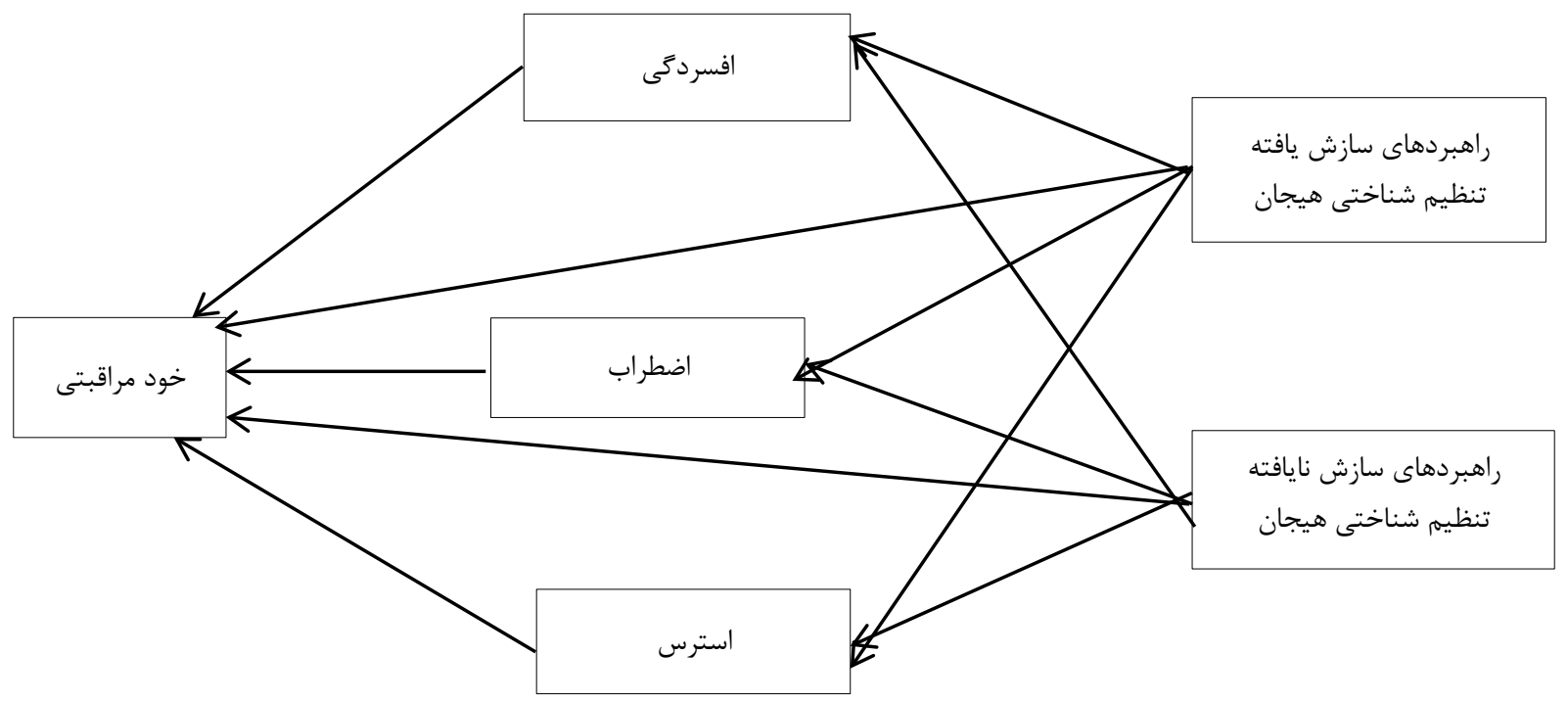

شكل ا: مسيرهاى مستقيم براى مدل فرضى نقش واسطه اى افسردى و اضطراب و استرس در رابطه بين تنظيم شناختى هيجان و خود مراقبتى در بيماران ديابتى نوع دو 


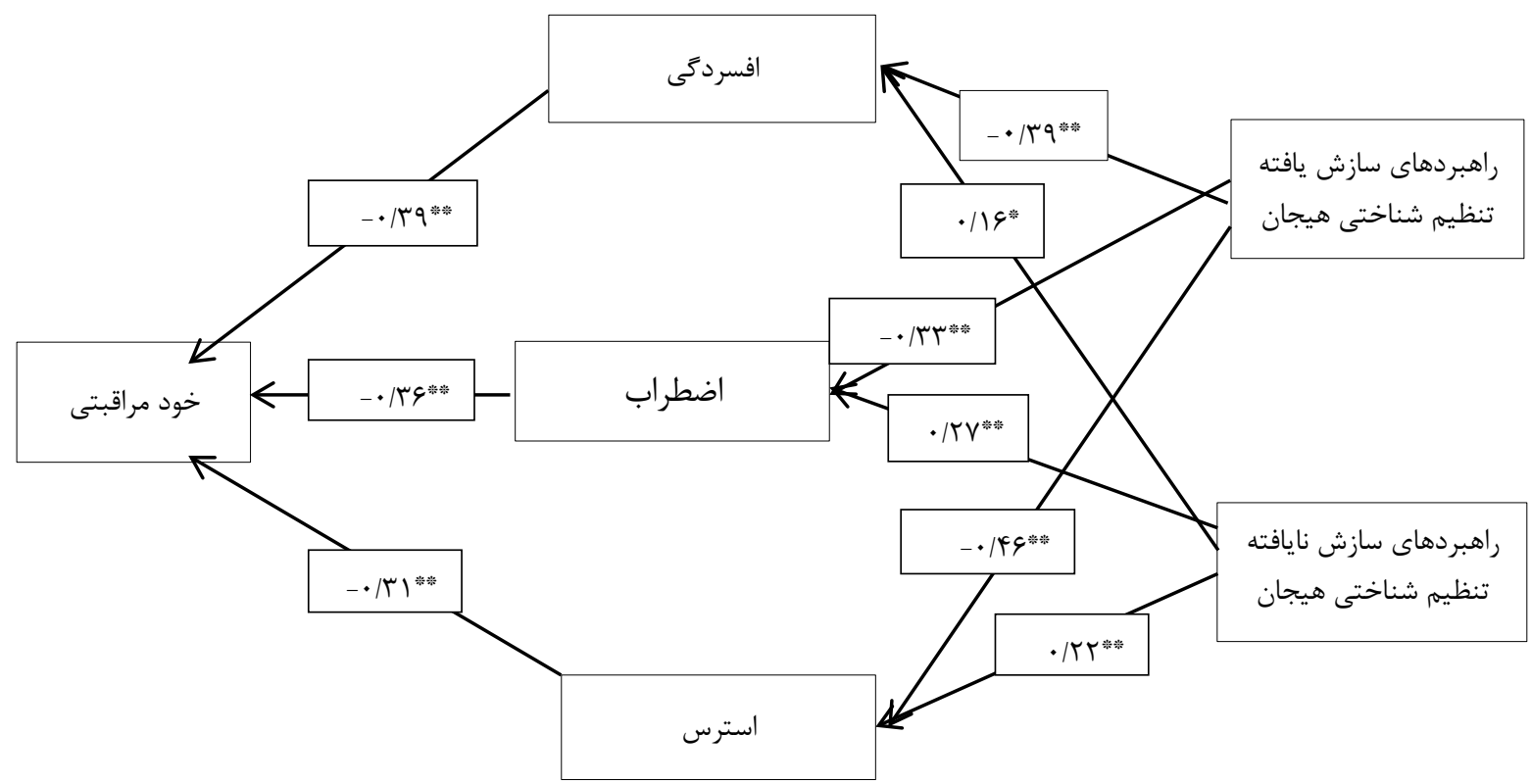

شكل ז: ضرايب استاندارد مسيرهاى مستقيم براى مدل فرضى نقش واسطه اى افسردىى و اضطراب و استرس در رابطه بين تنظيم شناختى هيجان و

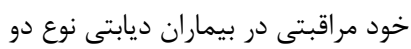

جدول ז: ضرايب استاندارد و غير استاندارد مسيرهاى مستقيم مدل فرضى نقش واسطه اى افسردگى و اضطراب و استرس در رابطه بين تنظيم شناختى هيجان و خود مراقبتى در بيماران ديابتى نوع دو مدئى

\begin{tabular}{|c|c|c|c|}
\hline $\mathbf{p}$ & $\beta$ & B & مسير ها \\
\hline$\cdot 1 \cdot r r$ & $-\cdot / \Gamma q^{* * * m}$ & $-\cdot /\left.1\right|^{\text {蒡 }}$ & اثر راهبردهاى سازش يافته تنظيمى هيجان بر افسردىى \\
\hline$\cdot / \cdot \Delta$ & $-\cdot \mid \uparrow 4^{* * *}$ & $-\cdot / 19^{* * *}$ & اثر راهبردهاى سازش يافته تنظيم هيجان بر استرس \\
\hline .1 .99 & $-\cdot / \mu r^{\text {絭 }}$ & $-\cdot / 1 \cdot{ }^{* * *}$ & اثر راهبردهاى سازش يافته تنظيم هيجان بر اضطراب \\
\hline$\cdot / \cdot \varphi$ & $\cdot 119^{*}$ & $\cdot / 11^{*}$ & اثر راهبردهاى سازش نايافته تنظيم هيجان بر افسردىى \\
\hline$\cdot / \cdot 1$ & $\cdot / T r^{* * * \%}$ & $\cdot /\left.1\right|^{\text {絭 }}$ & اثر راهبردهاى سازش نايافته تنظيم هيجان بر استرس \\
\hline $.1 \cdot 9$ & $\cdot / T V^{* * *}$ & $\cdot / 1 Q^{* * *}$ & اثر راهبردهاى سازش نايافته تنظيم هيجان بر اضطراب \\
\hline$\cdot 1 \cdot 1$ & $-\cdot / q^{* * * *}$ & $-1 / 11^{* * *}$ & اثر افسردگى بر خودمراقبتى \\
\hline $.1 . .4$ & $-\cdot /\left.\Gamma\right|^{\text {絭 }}$ & $-1 / \cdot V^{* * *}$ & اثر استرس بر خودمراقبتى \\
\hline$\cdot / \cdot v$ & $-\cdot / \varphi^{* * *}$ & $-1 / \Upsilon \varphi^{* *}$ & اثراضطراب بر خودمراقبتى \\
\hline
\end{tabular}

جدول با: ضرايب استاندارد مسيرهاى غير مستقيم مدل فرضى نقش واسطه اى افسردىى و اضطراب و استرس در رابطه بين تنظيم شناختى هيجان و

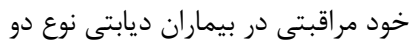

\begin{tabular}{|c|c|}
\hline $\boldsymbol{\beta}$ & 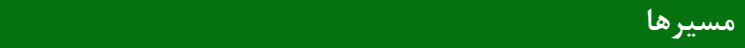 \\
\hline$\cdot|r|^{\text {***: }}$ & اثر غير مستقيم راهبردهاى سازش يافته تنظيم هيجان بر خودمراقبتى \\
\hline$-\cdot / 1 r^{\text {䋨 }}$ & اثر غيرمستقيم راهبردهاى سازش نايافته تنظيمم هيجان بر خودمراقبتى \\
\hline
\end{tabular}

جدول f: شاخص هاى برازش مدل فرضى نقش واسطه اي افسردى و اضطراب و استرس در رابطه بين تنظيم شناختى هيجان و خود مراقبتى در بيماران ديابتى نوع دو و

\begin{tabular}{|c|c|c|c|c|c|c|c|}
\hline CFI & AGFI & GFI & RMSEA & $\mathrm{X}^{2} / \mathrm{df}$ & p & df & $\mathrm{X}^{2}$ \\
\hline ./9V & .191 & .191 & $\cdot / \cdot r$ & $\Delta / G T$ & $\mathrm{P}>\cdot 1 \cdot 1$ & $\Delta$ & $r N / / F$ \\
\hline
\end{tabular}

نشان دهنده برازش مدل نسبت به مدل استقلال است و

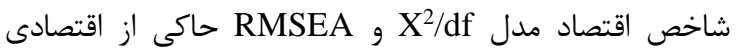

$$
\text { بودن آن است. }
$$

جنان جه مشاهده مى شود، شاخص هاى مدل حاكى از برازش

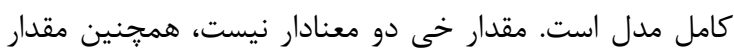
AGFI و مدل و شاخص مقايسهاى برازش مدل مطلوبند. 
خود اجتناب مىكنند و در بيان هيجانات خود به صورت

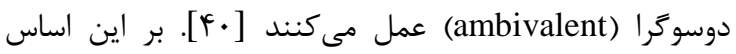

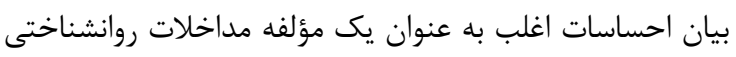

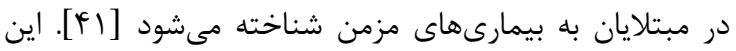

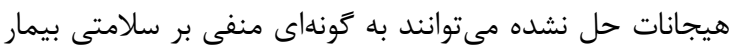

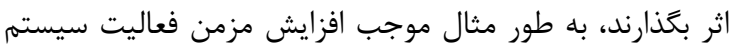

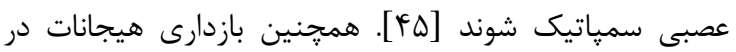

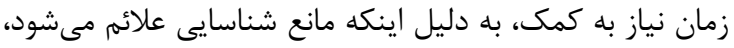

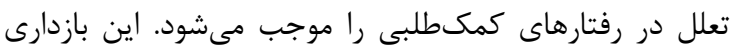

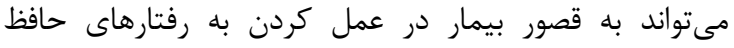

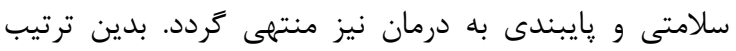

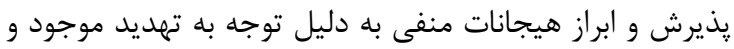

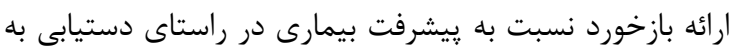

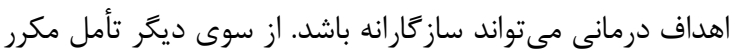

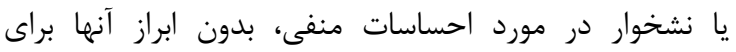

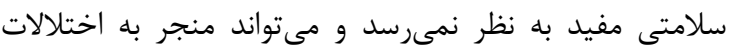

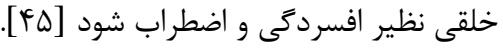

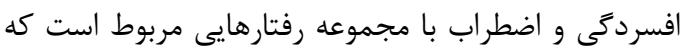

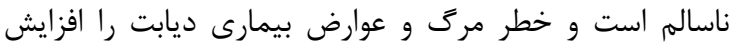

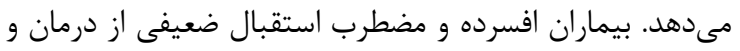

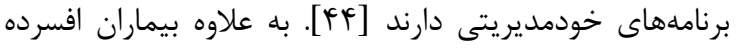

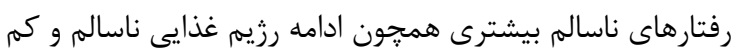

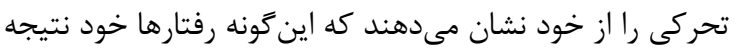

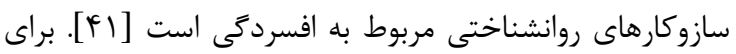

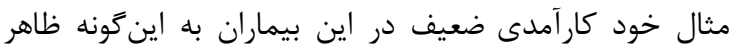

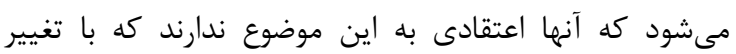

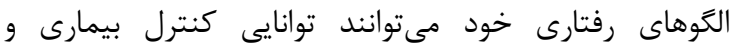

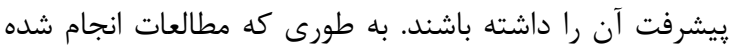

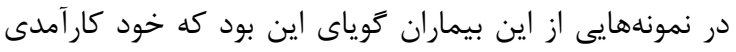

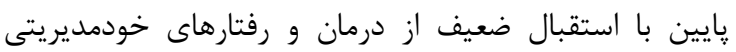

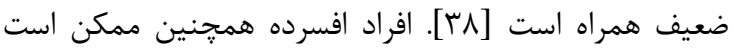

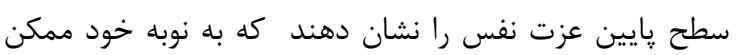

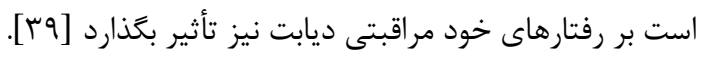

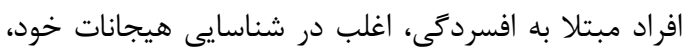

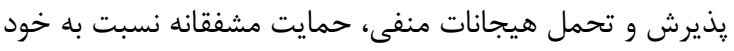

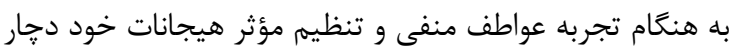

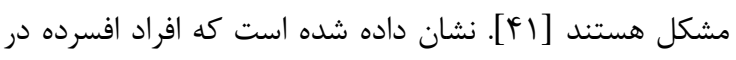

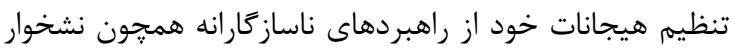

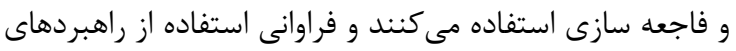

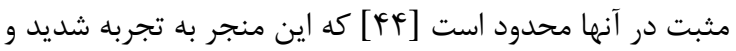

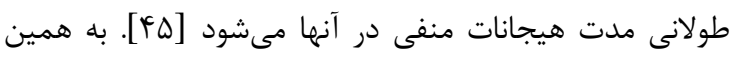

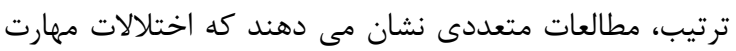

نتايج يزوهش نشان داد كه در مبتلايان به ديابت نوع دو، خودمراقبتى با راهبردهاى سازش يافته تنظيم شناختى هيجان

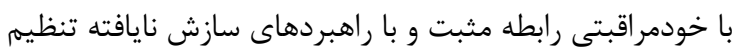

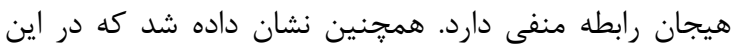

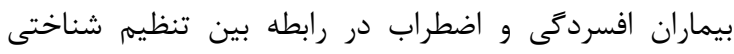

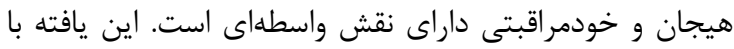

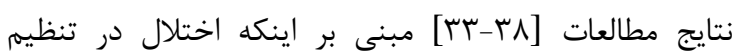

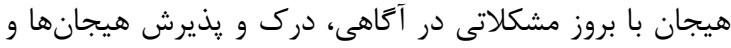

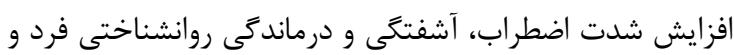

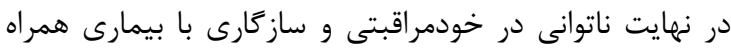
است، همسو مىباشد. در تبيين جنين نتايجى بايد بيان داشت كه تنظيم

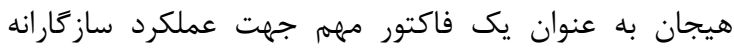

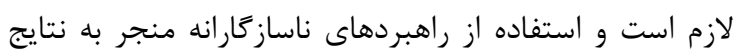

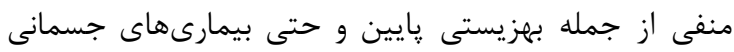

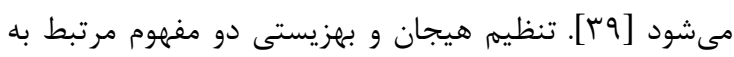

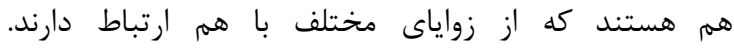

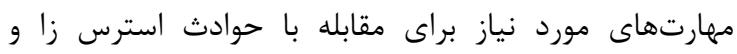

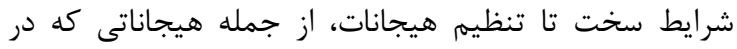

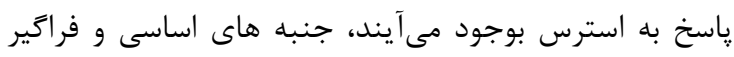

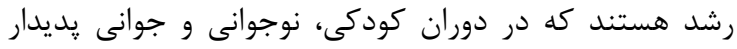

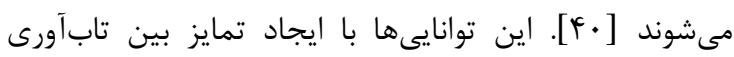

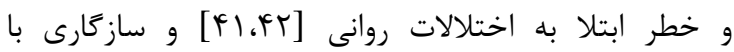

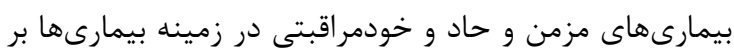

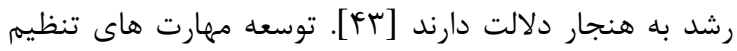

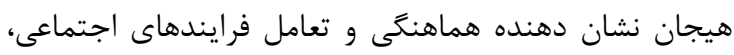

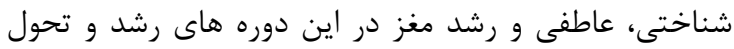

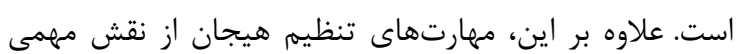

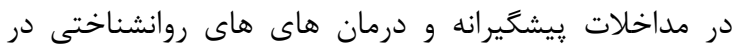

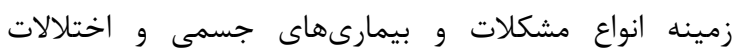

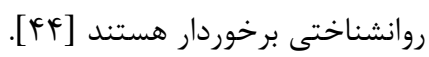

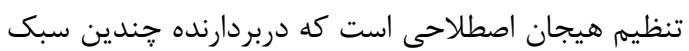

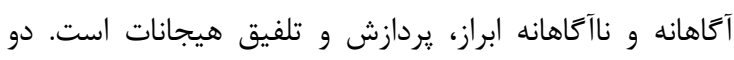

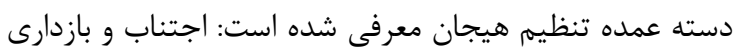

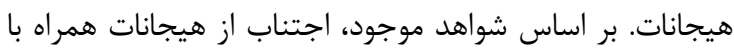

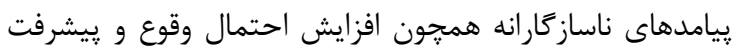

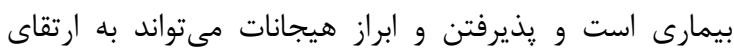

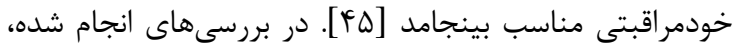

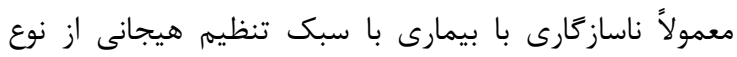

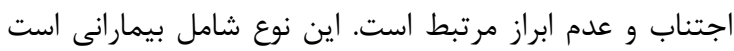

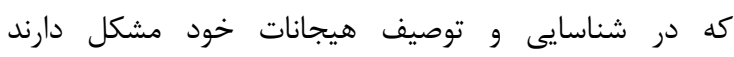

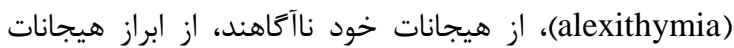




$$
\begin{aligned}
& \text { تنظيم هيجان سازش يافته استفاده مىكنند داراى اين توانايى }
\end{aligned}
$$

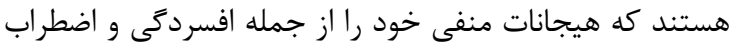

$$
\begin{aligned}
& \text { كنترل كرده و و بدين طريق بر اين بيمارى فائق آمده و فرايند }
\end{aligned}
$$

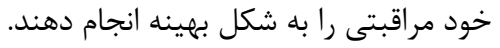

$$
\begin{aligned}
& \text { تشكر و قرر فاذى } \\
& \text { نويسندكان اين مقاله بر خود لازم مى دادانيد، از حمايتهاى }
\end{aligned}
$$

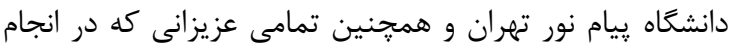

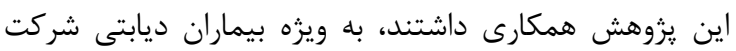

$$
\begin{aligned}
& \text { كننده كمال تشكر و قدردانى نمايند. }
\end{aligned}
$$$$
\text { تضاد منافع }
$$$$
\text { اين مطالعه براى نويسندكان هيج گَونه تضاد منافعى نداشته }
$$

\section{REFERENCES}

1. Akushevich I, Yashkin AP, Kravchenko J, Fang F, Arbeev $\mathrm{K}$, Sloan F, Yashin AI. Identifying the Causes of the Changes in the Prevalence Patterns of Diabetes in Older US Adults: A New Trend Partitioning Approach. Journal of Diabetes and its Complications. 2018. doi.org/10.1016/j. jdiacomp.2017.12.014

2. Shah AD, Rushakoff RJ. Patient self-management of diabetes care in the inpatient setting: con. Journal of diabetes science and technology. 2015;9(5):1155-7. doi.org/10.1177/1932296815586581

3. Khan AR, Al Abdul Lateef ZN, Khamseen MB, Al Aithan MA, Khan SA, Al Ibrahim I. Knowledge, attitude and practice of ministry of health primary health care physicians in the management of type 2 diabetes mellitus: A crosssectional study in the Al Hasa District of Saudi Arabia. Niger J Clin Pract. 2011; 14: 52-9.

4. Powers MA, Bardsley J, Cypress M, Duker P, Funnell MM, Fischl AH, Maryniuk MD, Siminerio L, Vivian E. Diabetes self-management education and support in type 2 diabetes: a joint position statement of the American Diabetes Association, the American Association of Diabetes Educators, and the Academy of Nutrition and Dietetics. Journal of the Academy of Nutrition and Dietetics. 2015;115(8):1323-34. doi.org/10.1177/0145721716689694

5. Ilanne-Parikka P, Eriksson JG, Lindström J, Peltonen M, Aunola S, Hämäläinen $\mathrm{H}$, Keinänen-Kiukaanniemi S, Laakso M, Valle TT, Lahtela J, Uusitupa M. Effect of lifestyle intervention on the occurrence of metabolic syndrome and its components in the Finnish Diabetes Prevention Study. Diabetes care. 2008;31(4):805 -7.doi. org/10.2337/dc07-1117

6. DeCoster VA. Challenges of type 2 diabetes and role of health care social work: A neglected area of practice. Health \& Social Work. 2001;26(1):26-37.

7. Elder NC, Muench J. Diabetes care as public health. Journal of Family Practice. 2000;49(6):513-.521. PMID:11338276

8. Young-Hyman D, De Groot M, Hill-Briggs F, Gonzalez JS, Hood K, Peyrot M. Psychosocial care for people with diabetes: a position statement of the American Diabetes Association. Diabetes Care. 2016;39(12):2126-40. doi.org/ 10.2337/dc16-2053

9. Schinckus L, Dangoisse F, Van den Broucke S, Mikolajczak M. When knowing is not enough: Emotional distress and depression reduce the positive effects of health literacy on diabetes self-management. Patient education and counseling. 2018;101(2):324-30. doi: 10.1016/j.pec.

$$
\begin{aligned}
& \text { هاى تنظيم هيجان در بروز و حفظ اختلالات اضطرابى نيز نقش }
\end{aligned}
$$

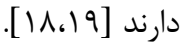

$$
\begin{aligned}
& \text { از جمله محدوديتهاى يثوهش حاضر مى توان به } \\
& \text { همبستخى بودن طرح يزوهش آن اشاره كرد كه اسنادهاى علت } \\
& \text { شناختى را در تفسير يافتهها محدود مى سازد. از ديگر } \\
& \text { محدوديت ها، عدم به كارگيرى عوامل بيشتر در نقش متغير } \\
& \text { مستقل و واسطه اى و بررسى وضعيت آنها در ييش بينى خود } \\
& \text { مراقبتى در بيماران ديابتى بود.ا بنابراين توصيه مى شود در } \\
& \text { مطالعات بعدى به بررسى نقش متغيرهاى بيشتر در پيش بينى } \\
& \text { خود مراقبتى در بيماران ديابتى يرداخته شود. }
\end{aligned}
$$

\section{نتيجه كيرى}

نتايج يزوهش حاضر نشان داد راهبردهاى تنظيم هيجان از

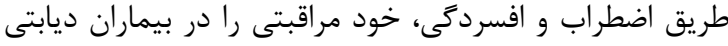

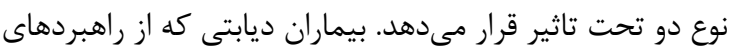

10. Calhoun D, Beals J, Carter EA, Mete M, Welty TK, Fabsitz RR, Lee ET, Howard BV. Relationship between glycemic control and depression among American Indians in the Strong Heart Study. Journal of Diabetes and its Complications. 2010;24(4):217-22. doi:10.1016/j.jdiacomp. 2009.03.005

11. Gonzalez JS, Safren SA, Cagliero E, Wexler DJ, Delahanty L, Wittenberg E, Blais MA, Meigs JB, Grant RW. Depression, self-care, and medication adherence in type 2 diabetes: relationships across the full range of symptom severity. Diabetes care. 2007;30(9):2222-7. doi: 10.2337/ dc07-0158

12. Thompson RA. Emotion and emotion regulation: Two sides of the developing coin. Emotion Review. 2011;(1):53-61. doi: 10.1177/1754073910380969

13. Garnefski N, Kraaij V. Relationships between cognitive emotion regulation strategies and depressive symptoms: A comparative study of five specific samples. Personality and Individual differences. 2006;40(8):1659-69. doi:10.1016/j. paid.2005.12.009

14. Tice DM, Bratslavsky E. Giving in to feel good: The place of emotion regulation in the context of general self-control. Psychological Inquiry. 2000;11(3):149-59.

15. Bargh JA, Williams LE. The nonconscious regulation of emotion. Handbook of emotion regulation. 2007; 1:429Á445. doi:10.1037/a0017745

16. Carl JR, Soskin DP, Kerns C, Barlow DH. Positive emotion regulation in emotional disorders: a theoretical review. Clin Psychol Rev. 2013;33(3):343- 60. doi: 10.1016/j.cpr. 2013.01.003.

17. Campbell-Sills L, Barlow DH. Incorporating emotion regulation into conceptualizations and treatments of anxiety and mood disorders. In: Gross JJ, editor. Handbook of Emotion Regulation. New York: Guilford Press; 2007:542559. doi: 10.4236/jss.2016.42020

18. Hofmann SG, Sawyer AT, Fang A, Asnaani A. Emotion dysregulation model of mood and anxiety disorders. Depression and anxiety. 2012;29(5):409-16. doi: 10.1002/ da. 21888

19. Aldao A, Nolen-Hoeksema S. When are adaptive strategies most predictive of psychopathology?. Journal of abnormal psychology. 2012;121(1):276. doi: 10.1037/a0023598

20. Siegel EY, Haller M, Cui R, Trim RS, Tate SR, Norman SB. Examining changes in negative mood regulation expectancies, posttraumatic stress disorder, depression, and substance use following integrated cognitive-behavioral 
therapy. Substance abuse. 2017;38(4):468-72. doi: 10.1080/ 08897077.

21. Ehring T, Tuschen-Caffier B, Schnülle J, Fischer S, Gross JJ. Emotion regulation and vulnerability to depression: Spontaneous versus instructed use of emotion suppression and reappraisal. Emotion. 2010; 10(4):563-572. doi: 10.1037/a0019010

22. Berking M, Wirtz CM, Svaldi J, Hofmann SG. Emotion regulation predicts symptoms of depression over five years. Behaviour research and therapy. 2014;57:13-20. PMID: 27231404

23. Pollock NC, McCabe GA, Southard AC, Zeigler-Hill V. Pathological personality traits and emotion regulation difficulties. Personality and Individual Differences. 2016;95:168-77. doi:10.5964/ejop.v12i3.1109

24. Gross JJ. Emotion regulation: taking stock and moving forward. Emotion. 2013;13(3):359. doi: 10.1037/a0032135.

25. Gross JJ, Muñoz RF. Emotion regulation and mental health. Clinical psychology: Science and practice. 1995;2(2):151-64.

26. Toobert DJ, Hampson SE, Glasgow RE. The summary of diabetes self-care activities measure: results from 7 studies and a revised scale. Diabetes Care. 2000; 23(7): 943-50. PMID: 10895844

27. Vosoghi Karkazloo N, Abootalebi Daryasari G, Farahani B, Mohammadnezhad E, Sajjadi A. The study of self-care agency in patients with diabetes (Ardabil). Mod Care J. 2012; 8(4) :197-204. (Persian)

28. Sorani M, Taghdisi MH, Shojaei Zadeh D, Novin L, Noroozi M, Fallahi S. Predictorsof Self-Care Behaviors of Patients with Type 2 Diabetes. Journal of Health System Research. 2013; 8(5):814-23. (Persian) doi:10.19082/4207

29. Crawford JR, Henry JD. The Depression Anxiety Stress Scales (DASS): Normative data and latent structure in a large non-clinical sample. British Journal of Clinical Psychology. 2003;42(2):111-131. doi: 10.1348/01446650 3321903544

30. Antony MM, Bieling PJ, Cox BJ, Enns MW, Swinson RP. Psychometric properties of the 42- item and 21-item versions of the Depression Anxiety Stress Scales in clinical groups and a community sample. Psychological Assessment 1998;10(2):176-81.

31. Samani S, jokar B. Evaluate the reliability and validity of the short form of depression, anxiety and stress. J Social Sci Humanities Shiraz Univ 2007; 26 (3): 65-76.

32. Hasani J. The reliability and validity of the short form of the cognitive emotion regulation questionnaire. J Res Behav Sci. 2011; 9(4): 229-40. (Persian)

33. Gratz KL, Roemer L. Multidimensional assessment of emotion regulation and dysregulation: Development, factor structure, and initial validation of the Difficulties in Emotion
Regulation Scale. Journal of Psychopathology and Behavioral Assessment, 2004;26: 41-54. doi: 10.1007/ s10862-008-9102-4

34. Thompson RA, Meyer S. Socialization of emotion regulation in the family. Handbook of emotion regulation. 2007;249:249-68.

35. Kneeland ET, Dovidio JF, Joormann J, Clark MS. Emotion malleability beliefs, emotion regulation, and psychopathology: Integrating affective and clinical science. Clinical psychology review. 2016;45:81-8. doi: 10.1016/j.

36. Mennin D, Farach F. Emotion and evolving treatments for adult psychopathology. Clinical Psychology: Science and Practice. 2007;14(4):329-52.

37. Hofmann SG, Grossman P, Hinton DE. Loving-kindness and compassion meditation: Potential for psychological interventions. Clinical psychology review. 2011;31(7):112632. doi: 10.1016/j.cpr.2011.07.003

38. Hofmann SG, Sawyer AT, Fang A, Asnaani A. Emotion dysregulation model of mood and anxiety disorders. Depression and anxiety. 2012;29(5):409-16. doi: 10.1155/ 2015/269126

39. Gross JJ, Muñoz RF. Emotion regulation and mental health. Clinical psychology: Science and practice. 1995;2(2):15164. doi.org/10.1111/j.1468-2850.1995.tb00036.x

40. Denollet J, Nykliček I, Vingerhoets AJ. Introduction: Emotions, emotion regulation, and health. InEmotion regulation 2008 (pp. 3-11). Springer, Boston, MA.

41. Compas BE, Jaser SS, Dunn MJ, Rodriguez EM. Coping with chronic illness in childhood and adolescence. Annual review of clinical psychology. 2012;8:455-80. doi: 10.1037/ hea0000083

42. Compas BE, Watson KH, Reising MM, Dunbar JP. Stress and coping: Transdiagnostic processes in child and adolescent psychopathology. Transdiagnostic mechanisms and treatment approaches of youth psychopathology. 2013:35-58

43. Cole PM, Martin SE, Dennis TA. Emotion regulation as a scientific construct: Methodological challenges and directions for child development research. Child development. 2004;75(2):317-33. doi: 10.1111/j.17508606.2008.00056.x

44. Mennin DS, Ellard KK, Fresco DM, Gross JJ. United we stand: Emphasizing commonalities across cognitivebehavioral therapies. Behavior Therapy. 2013;44(2):234-48. doi:10.1016/j.beth.2013.02.004

45. Austenfeld JL, Stanton AL. Coping through emotional approach: a new look at emotion, coping, and health-related outcomes. Journal of personality. 2004;72(6):1335-64. doi:10.1111/j.1467-6494.2004.00299.x 